\title{
Development of measurement-based load models for the dynamic simulation of distribution grids
}

\author{
Eleftherios O. Kontis, Mazheruddin H. Syed, Efren Guillo-Sansano Theofilos A. Papadopoulos, \\ Andreas I. Chrysochos, Grigoris K. Papagiannis, Graeme M. Burt
}

\begin{abstract}
The advent of new types of loads, such as power electronics and the increased penetration of low-inertia motors in the existing distribution grids alter the dynamic behavior of conventional power systems. Therefore, more accurate dynamic, aggregate, load models are required for the rigorous assessment of the stability limits of modern distribution networks. In this paper, a measurement-based, input/output, aggregate load model is proposed, suitable for dynamic simulations of distribution grids. The new model can simulate complex load dynamics by employing variable-order transfer functions. The minimum required model order is automatically determined through an iterative procedure. The applicability and accuracy of the proposed model are thoroughly evaluated under distinct loading conditions and network topologies using measurements acquired from a laboratory-scale test setup. Furthermore, the performance of the proposed model is compared against other conventional load models, using the mean absolute percentage error.
\end{abstract}

Index Terms-Distribution grids, dynamic equivalencing, measurement-based approach, system identification techniques.

\section{INTRODUCTION}

Despite the research efforts from both academia and industry, modeling of distribution grids remains a very challenging task [1]. The vast number of different individual electric and electronic devices in distribution networks and their timevarying, stochastic nature pose several difficulties in the already complex modeling procedure [2]. To overcome these issues, aggregated load models are typically adopted by system operators [3].

The load modeling challenge is to determine an equivalent representation for the aggregation of different types of individual components, supplied by a common busbar [4], [5].

The work in this paper has been supported by the European Commission, under the FP7 project ELECTRA (grant no: 609687) and Horizon 2020 project ERIGrid (grant no: 654113). Any opinions, findings, and conclusions or recommendations expressed in this material are those of the authors and do not necessarily reflect those of the European Commission.

Eleftherios O. Kontis, and Grigoris K. Papagiannis are with the Power Systems Laboratory, School of Electrical and Computer Engineering, Aristotle University of Thessaloniki, Thessaloniki, Greece, GR 54124, (e-mail: kontislefteris@gmail.com; grigoris@eng.auth.gr).

Theofilos A. Papadopoulos is with the Power Systems Laboratory, Dept of Electrical and Computer Engineering, Democritus University of Thrace, Xanthi, Greece, GR 67100, (e-mail: thpapad@ee.duth.gr).

Andreas I. Chrysochos is with the Cablel ${ }^{\circledR}$ Hellenic Cables S.A., Viohalco Group, Sousaki Korinthias, Korinthos, Greece, GR 20100 (e-mail: achrysochos@fulgor.vionet.gr).

Mazheruddin H. Syed, Efren Guillo-Sansano, and Graeme M. Burt are with the Institute for Energy and Environment University of Strathclyde, Glasgow, UK (e-mail: mazheruddin.syed@strath.ac.uk; efren.guillosansano@strath.ac.uk; graeme.burt@strath.ac.uk).
Generally, load modeling procedure consists of two dinstinct stages. In the first stage, a suitable load model structure is defined, while in the second stage the required model parameters are estimated [3].

The choice of the model structure depends on the needs of the analysis, the expected accuracy, and the load composition. Therefore, load models are divided into two main categories: Static and dynamic models [2], [3]. Static load models describe the relationship between load real/reactive power, voltage and/or frequency at any time instant using algebraic equations. Static models can be used for loads that do not exhibit significant dynamic response after a disturbance [6] or when analysis of the equilibrium conditions is only considered [7]. In case of voltage and angular stability analysis, dynamic load models are required. Dynamic models describe the relationship between load real/reactive power at any time instant as functions of voltage and/or frequency of the present and past time instants [8]. Difference or differential equations are used to describe such models.

Once the model structure is specified, the remaining task focuses on the estimation of the required model parameters. For this purpose, the component- and the measurement-based approaches can be used. The implementation of componentbased methodology requires reliable information of the load class mix, the load components as well as a priori knowledge of typical characteristics of individual devices [8]. Therefore, the application of this method requires accurate data, which usually cannot be determined in distribution networks due to their size and confidentiality [9]. On the other hand, in the measurement-based approach the required model parameters are estimated from in-situ measurements, using parameter identification techniques [2], [10]. This method directly captures the actual load dynamics, resulting in more accurate models. Moreover, when new measurements are available, the required model parameters can be easily updated close to realtime, enhancing the accuracy of the developed models [6], [8]. The aforementioned advantages in conjunction with the increased availability of measurements due to the installation of phasor measurement units (PMUs) at distribution level, constitute the measurement-based approach more appealing compared to the component-based methodology [11].

In this paper, a measurement-based, aggregate, load model is proposed, suitable for the dynamic simulation of distribution grids. The proposed model adopts the input/output structure of the well-established exponential recovery load model (ERLM) [12], [13]. However, contrary to the conventional ERLM, the 
new model employs variable-order transfer functions for the modeling of the recovery phase of the load. Thus, it can reproduce accurately complex dynamic phenomena caused by power electronic loads and motor drives. The accuracy and effectiveness of the proposed model are evaluated using measurements acquired from a laboratory-scale test setup. Furthermore, its performance is thoroughly compared with other conventional load models.

Following this introduction, the remaining of the paper is organized as follows: In Section II, an overview of the ERLM is presented. The mathematical formulation of the proposed dynamic load model and the corresponding parameter estimation procedure are explained in Section III. Section IV describes the examined laboratory setup. The performance of the proposed model is evaluated in Section V, while in Section VI sensitivity analysis is performed. Finally, Section VII concludes the paper.

\section{TheOreticAl BACKGROUND}

The general dynamic response of conventional power system loads is presented in Fig. 1. As shown, immediately after a voltage disturbance, the load consumption decreases instantaneously to $y_{+}$value. After the transient overshoot a recovery phase occurs. During this phase the load demand gradually recovers to a new steady-state value, i.e. to $y_{s s}$.

To simulate this dynamic behavior, Hill and Karlsson proposed the use of the ERLM [12], [13], the mathematical representation of which is defined as:

$$
\begin{gathered}
T_{y} \dot{y}_{r}(t)+y_{r}(t)=y_{s}(t)-y_{t}(t) \\
y_{l}(t)=y_{r}(t)+y_{t}(t)
\end{gathered}
$$

where $y_{l}$ denotes the total load demand in real or reactive power at time $t, y_{r}$ is the recovery state of the load, $y_{s}$ and $y_{t}$ are two auxiliary functions describing the steady-state and transient characteristics of the load, respectively. These functions are defined as:

$$
y_{s}(t)=y_{0}\left[\frac{V_{L}(t)}{V_{0}}\right]^{a_{s}}
$$
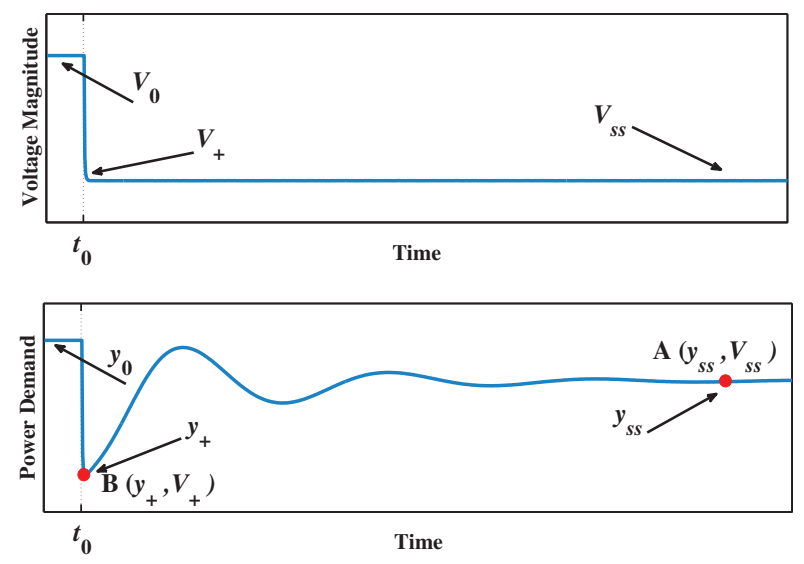

Fig. 1. Typical response of conventional power system loads.

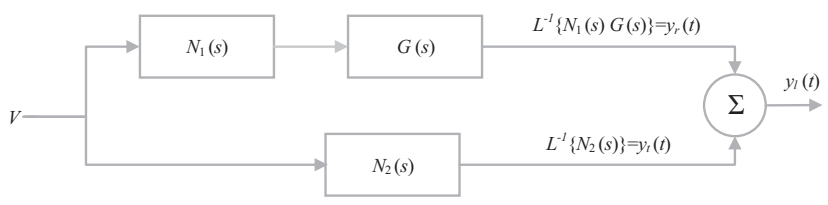

Fig. 2. Block diagram representation of the ERLM.

$$
y_{t}(t)=y_{0}\left[\frac{V_{L}(t)}{V_{0}}\right]^{a_{t}}
$$

Where $V_{0}$ and $y_{0}$ denote the voltage magnitude and power consumption prior to the disturbance as shown in Fig. 1, $V_{L}(t)$ is the measured load voltage, $a_{s}$ and $a_{t}$ are the steady-state and transient voltage exponents, respectively. The values of $a_{s}$ and $a_{t}$ can be calculated from the operating points $A\left(y_{s s}, V_{s s}\right)$ and $B\left(y_{+}, V_{+}\right)$, using the following algebraic equations:

$$
a_{s}=\frac{\log \left(\frac{y_{s s}}{y_{0}}\right)}{\log \left(\frac{V_{s s}}{V_{0}}\right)} \quad a_{t}=\frac{\log \left(\frac{y_{+}}{y_{0}}\right)}{\log \left(\frac{V_{+}}{V_{0}}\right)}
$$

By introducing the following simplifications:

$$
\begin{gathered}
N_{1}(t)=y_{s}(t)-y_{t}(t) \\
N_{2}(t)=y_{t}(t)
\end{gathered}
$$

Eqs. (1) and (2) can be rewritten as:

$$
\begin{gathered}
T_{y} \dot{y}_{r}(t)+y_{r}(t)=N_{1}(t) \\
y_{l}(t)=y_{r}(t)+N_{2}(t)
\end{gathered}
$$

By applying the Laplace transform and performing some simple manipulations, (8) and (9) can be rewritten as:

$$
\begin{gathered}
y_{r}(s)=N_{1}(s) \cdot \frac{1 / T_{y}}{s+1 / T_{y}} \\
y_{l}(s)=N_{2}(s)+N_{1}(s) \cdot \frac{1 / T_{y}}{s+1 / T_{y}}
\end{gathered}
$$

The block structured representation of (10) and (11) is presented in Fig. 2. As shown, the ERLM can be perceived as a block diagram interconnection of two nonlinear, exponential functions and a first-order linear transfer function. The gain of this transfer function is equal to $1 / T_{y}$, while the corresponding pole is $-1 / T_{y}$.

\section{Proposed Equivalent Model}

The increased number of power system loads, integrated to distribution grids via power electronics, results in complex dynamic phenomena, in which the first-order ERLM fails to provide appropriate representation. To overcome this issue, in this paper, a variable-order, input/output, equivalent load model is formulated. Voltage disturbances are considered as the inputs to the model, while real and reactive power responses are the corresponding outputs. 
To develop the proposed model, the block diagram representation of Fig. 2 is extended by employing high-order linear transfer functions, with general form as:

$$
G(s)=\frac{\beta_{\nu} s^{\nu}+\beta_{\nu-1} s^{\nu-1}+\ldots+\beta_{0}}{s^{\mu}+\alpha_{\mu-1} s^{\mu-1}+\ldots+\alpha_{0}}=\sum_{m=1}^{\mu} \frac{c_{m}}{s-p_{m}}
$$

In this case, the only restriction is that the $G(s)$ function must be strictly proper, i.e., $\nu<\mu$, to ensure that the recovery of the load is continuous [12], [13]. Generally, the required set of parameters $\boldsymbol{\theta}=[\boldsymbol{p}, \boldsymbol{c}]$ can be estimated using parameter identification techniques [14], [15]. In this paper, $\theta$ is identified using the vector fitting (VF) method [16]-[18], and the optimal order of the employed transfer functions is determined using the iterative procedure of Algorithm 1.

When a voltage disturbance occurs, the resulting load and voltage responses are recorded and used for the estimation of the required model parameters. Initially, voltage exponents $a_{s}$ and $a_{t}$ are determined from (5). Afterwards, functions $N_{1}$ and $N_{2}$ are computed in time-domain (TD) using (6) and (7), respectively. Subsequently, the recovery response of the load, i.e. $y_{r}$, is calculated in TD using (2). The Laplace transform (LT) of signals $N_{1}$ and $y_{r}$ is calculated and the response of the characteristic transfer function $G(s)$ is extracted in the frequency domain $(\mathrm{FD})$ :

$$
G(s)=\frac{\mathcal{L}\left(y_{r}(t)\right)}{\mathcal{L}\left(N_{1}(t)\right)}
$$

This response is approximated with a linear transfer function, denoted as $\hat{G}(s)$. To determine the minimum required order of $\hat{G}(s)$, the following iterative procedure, is adopted: In each iteration, the order of $\hat{G}(s)$ is increased by one and the required set of parameters $\boldsymbol{\theta}_{\boldsymbol{n}}$ is identified using the VF method. Here, $n$ denotes the $n$-th iteration of the algorithm and initially is set to one. Subsequently, as shown in Fig. 2, the estimated load response is calculated in TD using (14).

$$
y_{\text {est }}(t)=N_{2}(t)+\mathcal{L}^{-1}\left[N_{1}(s) \hat{G}(s)\right]
$$

Then, $y_{\text {est }}$ is compared with the actual load response $y_{l}$, with mean value $\bar{y}_{l}$, and the following validation index is calculated:

$$
R_{n}^{2}=\left(1-\frac{\sum_{m=1}^{M}\left(y_{l}[m]-y_{e s t}\left[m \mid \boldsymbol{\theta}_{n}\right]\right)^{2}}{\sum_{m=1}^{M}\left(y_{l}[m]-\bar{y}_{l}\right)^{2}}\right) \cdot 100 \%
$$

where $M$ denotes the total number of TD samples. Finally, the $\Delta R^{2}=R_{n}^{2}-R_{n-1}^{2}$ criterion, between the last two successful iterations is computed. For the first iteration, $R_{0}^{2}$ is set equal to zero. If the value of the $\Delta R^{2}$ coefficient is less than a predefined tolerance, the proposed modeling procedure terminates, resulting to a model order $n_{o}=n-1$ [19]. Otherwise, a higher order approximation for the $G(s)$ is computed.

\section{System UNDER STUdY}

To validate the applicability of the proposed model, a series of laboratory tests were conducted at the Dynamic Power

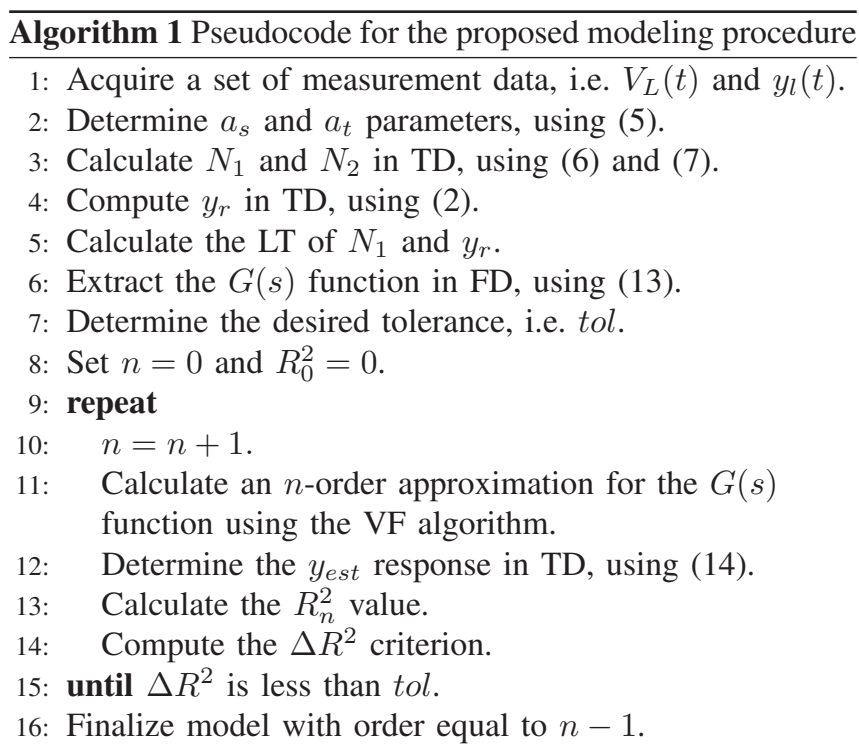

System Laboratory at University of Strathclyde. The test setup is comprised of a three-phase, 64-step, $10 \mathrm{kVA}$ static load bank (SLB) connected in parallel with three induction motors, as shown in Fig. 3. Using this setup, two different network topologies were examined by switching on and off switch $S_{1}$, respectively. Moreover, for each topology, five distinct load compositions were considered and examined by changing the nominal power of the SLB. Thus, as summarized in Table 1, a set of ten distinct network configurations were implemented and used to evaluate the performance of the proposed model.

The test setup is supplied by a three-phase programmable voltage source (PVS), allowing for instantaneous step-down voltage disturbances to be implemented. In all cases, a $-6 \%$ voltage disturbance was introduced and the corresponding voltage signals, the resulting real and reactive power responses at the point of common coupling (PCC) were recorded at a rate of 500 samples per second (sps). The acquired responses were used for the development and assessment of the proposed model.

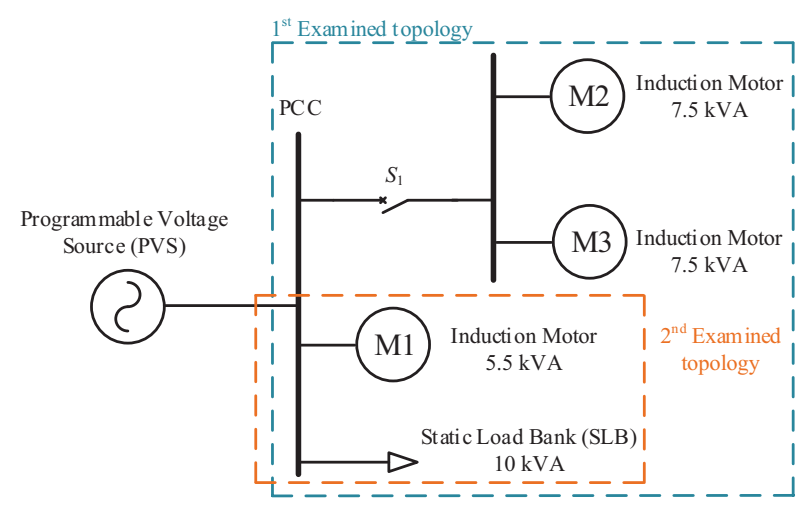

Fig. 3. Examined experimental setup. 
TABLE I

SYNOPSIS OF THE EXAMINED CONFIGURATIONS

\begin{tabular}{|c|c|c|c|c|}
\hline \multirow{2}{*}{$\begin{array}{c}\text { Examined } \\
\text { Configurations }\end{array}$} & SLB & M1 & M2 & M3 \\
\cline { 2 - 5 } C1.1 & $2.114 \mathrm{kw}, \mathrm{pf}=0.9$ & ON & ON & ON \\
\hline $\mathbf{C 1 . 2}$ & $4.077 \mathrm{kw}, \mathrm{pf}=0.9$ & ON & ON & ON \\
\hline $\mathbf{C 1 . 3}$ & $6.040 \mathrm{kw}, \mathrm{pf}=0.9$ & ON & ON & ON \\
\hline $\mathbf{C 1 . 4}$ & $8.003 \mathrm{kw}, \mathrm{pf}=0.9$ & ON & ON & ON \\
\hline $\mathbf{C 1 . 5}$ & $9.513 \mathrm{kw}, \mathrm{pf}=0.9$ & ON & ON & ON \\
\hline $\mathbf{C 2 . 1}$ & $2.114 \mathrm{kw}, \mathrm{pf}=0.9$ & ON & OFF & OFF \\
\hline $\mathbf{C 2 . 2}$ & $4.077 \mathrm{kw}, \mathrm{pf}=0.9$ & ON & OFF & OFF \\
\hline $\mathbf{C 2 . 3}$ & $6.040 \mathrm{kw}, \mathrm{pf}=0.9$ & ON & OFF & OFF \\
\hline $\mathbf{C 2 . 4}$ & $8.003 \mathrm{kw}, \mathrm{pf}=0.9$ & ON & OFF & OFF \\
\hline $\mathbf{C 2 . 5}$ & $9.513 \mathrm{kw}, \mathrm{pf}=0.9$ & ON & OFF & OFF \\
\hline
\end{tabular}

\section{Model Evaluation}

The accuracy of the proposed load model is thoroughly compared against the well-established ERLM, the exponential (EXP) [20] and the polynomial (ZIP) [20] load models, which are mostly used by distribution system operators for stability studies [21].

The parameters of the proposed model are estimated from the acquired measurements using the modeling procedure of Algorithm 1. On the other hand, the parameters for the conventional load models are determined using non-linear least square (NLS) optimization, targeting to minimize the following objective function [3].

$$
J(p)=\sum_{m=1}^{M}\left(y_{l}[m]-\hat{y}[m]\right)^{2}
$$

Where $M$ denotes the total TD samples, $p$ is the required set of parameters, while $\hat{y}[m]$ denotes the estimated load response at the $m$-th sample.

To quantify the accuracy of the examined load models, the mean absolute percentage error (MAPE (\%)) is used [7]:

$$
M A P E(\%)=\frac{100}{M} \sum_{m=1}^{M}\left|\frac{y_{l}[m]-\hat{y}[m]}{y_{l}[m]}\right|
$$

The calculated MAPEs for all examined configurations are summarized in Figs. 4 and 5.

The performance of the considered models is further analyzed in Figs. 6 and 7, where the measured real and reactive power responses are compared with the corresponding estimations, provided by the examined models.

In the first examined topology, after the power overshoot, caused by the instantaneous voltage drop, both real and reactive power recover to the new steady-state exponentially. On the other hand, in the second examined topology, both real and reactive power present an oscillatory behavior during the recovery period.

Based on the presented results it is clear that the EXP and ZIP load models fail to simulate adequately the dynamic
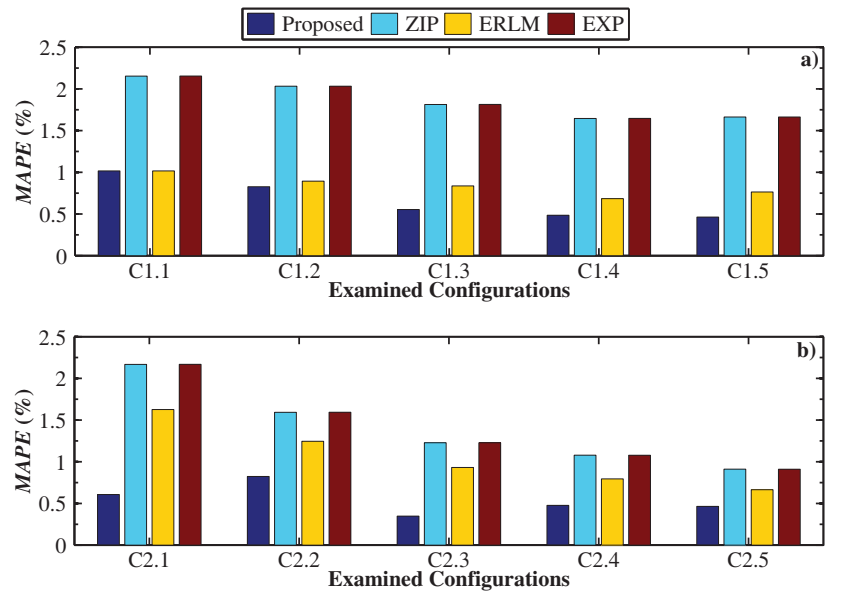

Fig. 4. MAPE (\%) for the modeling of the real power. Configurations derived from the a) first and b) second topology.
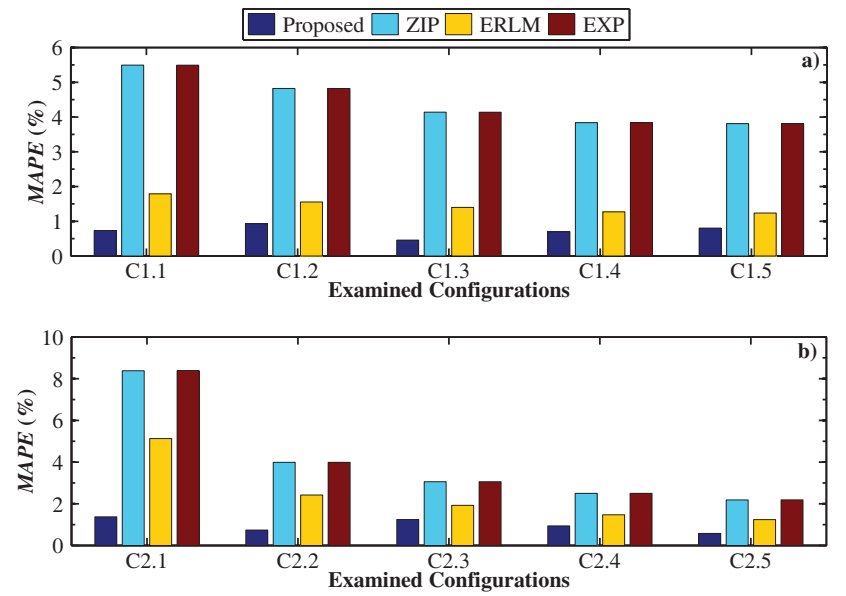

Fig. 5. MAPE (\%) for the modeling of the reactive power. Configurations derived from the a) first and b) second topology.

behavior of both real and reactive power. Indeed, due to the fact that both EXP and ZIP are static models, they can accurately estimate only the new steady-state power of the load. This is demonstrated in Figs. 6 and 7 and further verified by the corresponding MAPEs, depicted in Figs. 4 and 5 . Therefore, it can be concluded that these models are not appropriate to be used for the dynamic analysis of distribution grids.

On the other hand, the ERLM can describe with higher accuracy, compared to the ZIP and EXP models, the dynamic behavior of the load, since in all examined cases considerably lower MAPEs are calculated. Moreover, as shown in Figs. 6 and 7, the ERLM can estimate very accurately the new steadystate of both real and reactive power, capturing also adequately the power overshoot. However, the first-order ERLM cannot replicate the oscillatory behavior of the load.

On the contrary, the proposed load model presents the highest accuracy, as is evident from the very low MAPE values. Concerning the modeling of the real power, the corresponding 

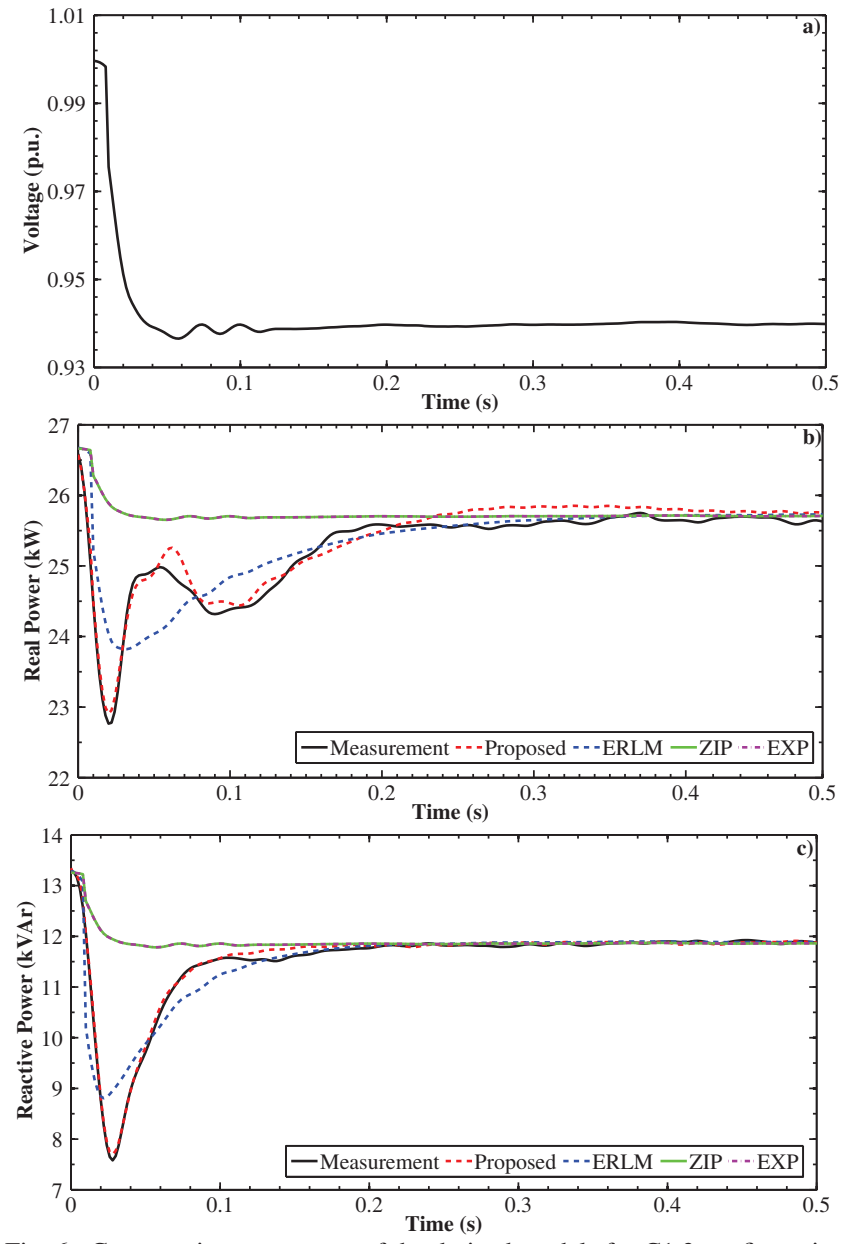

Fig. 6. Comparative assessment of the derived models for $\mathrm{C} 1.3$ configuration a) Examined voltage disturbance. Modeling of b) real power and c) reactive power.

MAPEs in all examined cases are lower than $1.02 \%$, while for the modeling of the reactive power MAPEs smaller that $1.38 \%$ are observed. The effectiveness of the proposed model is also qualitatively verified by the results depicted in Figs. 6 and 7. The developed model can simulate the dynamic behavior of both the real and reactive power, capturing accurately the overshoot, the recovery phase and the new steady-state of the load.

\section{SEnSitivity AnAlysis}

A sensitivity analysis is performed using the dynamic responses acquired from C1.3 and C2.3 configurations. Scope of the analysis is to investigate the influence of the model parameters on the accuracy of the simulated responses.

Initially, four discrete sets of optimal model parameters are determined using the proposed modeling procedure, describing the real and reactive power of $\mathrm{C} 1.3$ and $\mathrm{C} 2.3$ configurations. Next, sensitivity analysis is performed by introducing intentionally to each model parameter a $10 \%$ and $20 \%$ error from the original value. The resulting MAPEs are presented in Tables II and III. Higher MAPE values indicate higher
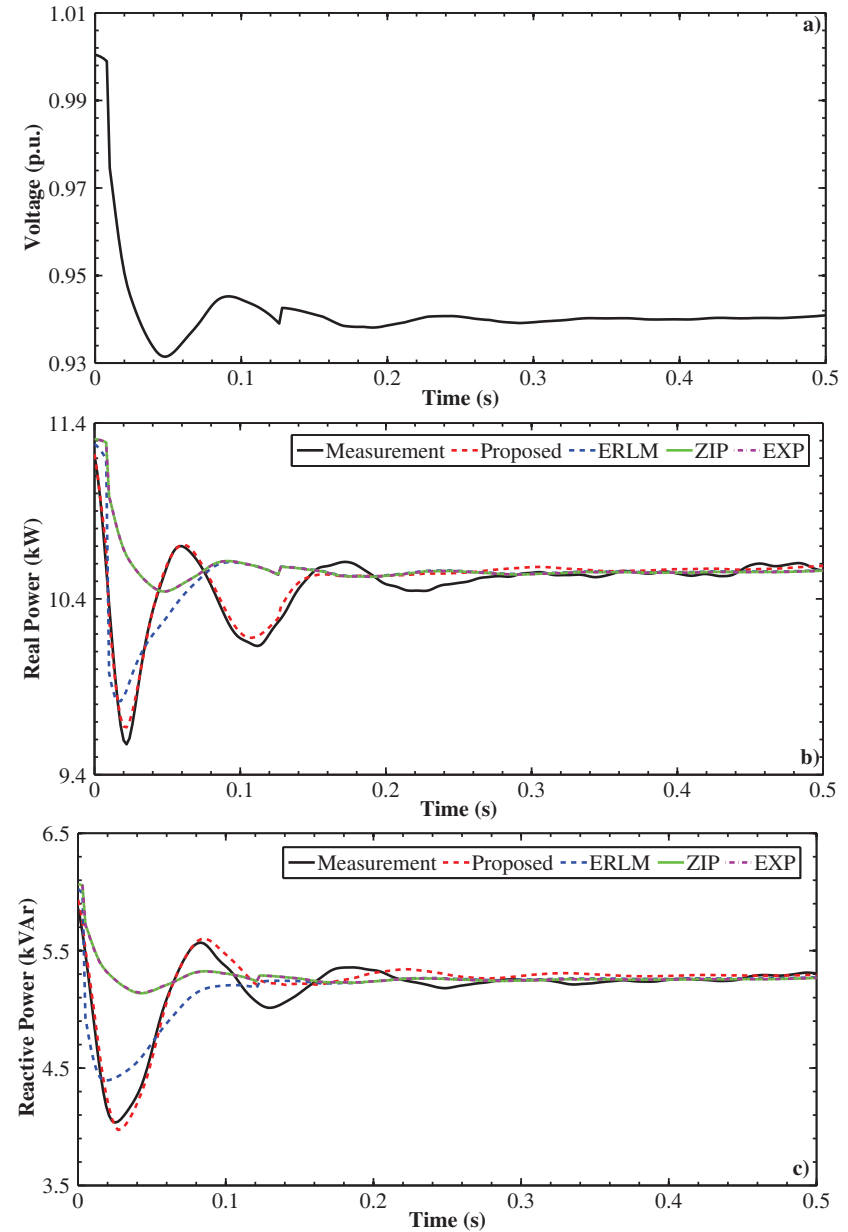

Fig. 7. Comparative assessment of the derived models for $\mathrm{C} 2.3$ configuration. a) Examined voltage disturbance. Modeling of b) real power and c) reactive power.

TABLE II

MAPES WITH VARIATION IN MODEL PARAMETERS. RESULTS FOR C1.3 CONFIGURATION.

\begin{tabular}{c|ccc|ccc}
\hline \multirow{2}{*}{} & \multicolumn{2}{c|}{ Modeling of real power } & \multicolumn{3}{c}{ Modeling of reactive power } \\
\cline { 2 - 7 } & \multicolumn{2}{c}{ Error in model parameters } & \multicolumn{2}{c}{ Error } & in model parameters \\
\cline { 2 - 7 } & $0 \%$ & $10 \%$ & $20 \%$ & $0 \%$ & $10 \%$ & $20 \%$ \\
\hline$a_{s}$ & 0.5527 & 0.7631 & 1.0231 & 0.4581 & 1.2117 & 2.0610 \\
$a_{t}$ & 0.5527 & 0.5841 & 0.7385 & 0.4581 & 0.6337 & 0.8477 \\
$\boldsymbol{p}$ & 0.5527 & 0.6299 & 0.8071 & 0.4581 & 0.7217 & 0.9692 \\
$\boldsymbol{c}$ & 0.5527 & 0.6361 & 0.8412 & 0.4581 & 0.5919 & 1.0550 \\
\hline
\end{tabular}

influence of the corresponding model parameter. Results show that erroneous values of the steady-state voltage exponent, i.e. $a_{s}$, result in the highest MAPEs, indicating the significant impact of this parameter on the model accuracy. Additionally, it is clear that the poles of the $G(s)$ function, i.e. $\boldsymbol{p}$, have also a significant effect on the model performance, while the transient voltage exponent, i.e. $a_{t}$, is the least influential parameter.

Finally, it is worth noticing that the proposed model provides more accurate results compared to the conventional 
TABLE III

MAPES WITH VARIATION IN MODEL PARAMETERS. RESULTS FOR C2.3 CONFIGURATION.

\begin{tabular}{c|ccc|ccc}
\hline \multirow{2}{*}{} & \multicolumn{3}{c|}{ Modeling of real power } & \multicolumn{3}{c}{ Modeling of reactive power } \\
\cline { 2 - 7 } & \multicolumn{3}{c}{ Error } & \multicolumn{3}{c}{ Error } \\
\cline { 2 - 7 } & $0 \%$ & $10 \%$ & $20 \%$ & $0 \%$ & $10 \%$ & $20 \%$ \\
\hline$a_{s}$ & 0.3477 & 0.4910 & 1.0865 & 1.2446 & 2.2734 & 3.5455 \\
$a_{t}$ & 0.3477 & 0.3565 & 0.4761 & 1.2446 & 1.4486 & 1.7397 \\
$\boldsymbol{p}$ & 0.3477 & 0.5462 & 0.7732 & 1.2446 & 1.8809 & 2.6196 \\
$\boldsymbol{c}$ & 0.3477 & 0.3765 & 0.5202 & 1.2446 & 1.3108 & 1.5688 \\
\hline
\end{tabular}

ZIP and EXP models even in cases where highly erroneous parameters are considered. This can be verified by the corresponding MAPE values, presented in Figs. 4 and 5. Specifically, concerning the modeling of real and reactive power for $\mathrm{C} 1.3$ configuration, both ZIP and EXP models result in MAPEs higher than $1.8 \%$ and $4.1 \%$, respectively, while for C2.3 configuration the corresponding MAPEs are higher than $1.23 \%$ and $3.06 \%$.

\section{CONCLUSIONS AND FUTURE WORK}

In this paper, a measurement-based, input/output, aggregate load model is proposed for the dynamic analysis of distribution systems. The proposed model is based on the wellestablished ERLM, however, contrary to the conventional firstorder ERLM, the proposed model uses variable-order linear transfer function to simulate accurately the recovery phase of the load.

The accuracy and applicability of the proposed model are thoroughly evaluated under different, distinct network configurations using measurements acquired from a laboratoryscale test setup. In all examined cases, the experimental results indicate that the developed model can capture very accurately the dynamic behavior of the load. Moreover, the performance of the proposed model is compared with the conventional ERLM, as well as with the static ZIP and EXP models. Additionally, a sensitivity analysis is performed to investigate the impact of the model parameters in the resulting accuracy. The sensitivity analysis in conjunction with the comparative assessment highlight the superior performance of the proposed model compared to the conventional approaches.

Future work will be conducted to validate the performance of the proposed model under different network configurations and voltage disturbances and to determine the most accurate system identification technique to estimate the parameters of the $G(s)$ function. Finally, the ability of the developed model to simulate the dynamic behavior of distribution networks with high penetration levels of distributed renewable energy sources will be also investigated.

\section{REFERENCES}

[1] D. P. Stojanovic, L. M. Korunovic, and J. Milanovic, "Dynamic load modelling based on measurements in medium voltage distribution network," Elect. Power Syst. Res., vol. 78, no. 2, pp. 228 - 238, Feb 2008.
[2] V. Knyazkin, C. A. Canizares, and L. H. Soder, "On the parameter estimation and modeling of aggregate power system loads," IEEE Trans. Power Syst., vol. 19, no. 2, pp. 1023-1031, May 2004.

[3] B.-K. Choi, H.-D. Chiang, Y. Li, H. Li, Y.-T. Chen, D.-H. Huang, and M. G. Lauby, "Measurement-based dynamic load models: derivation, comparison, and validation," IEEE Trans. Power Syst., vol. 21, no. 3 , pp. 1276-1283, Aug 2006.

[4] I. F. Visconti, D. A. Lima, J. M. C. d. S. Costa, and N. R. d. B. C. Sobrinho, "Measurement-based load modeling using transfer functions for dynamic simulations," IEEE Trans. Power Syst., vol. 29, no. 1, pp. 111120, Jan 2014.

[5] E. O. Kontis, A. I. Chrysochos, G. K. Papagiannis, and T. A. Papadopoulos, "Development of measurement-based generic load models for dynamic simulations," in 2015 IEEE Eindhoven PowerTech, June 2015, pp. 1-6.

[6] P. Regulski, D. S. Vilchis-Rodriguez, S. Djurovic, and V. Terzija, "Estimation of composite load model parameters using an improved particle swarm optimization method," IEEE Trans. Power Del., vol. 30 , no. 2, pp. 553-560, April 2015.

[7] A. Miranian and K. Rouzbehi, "Nonlinear power system load identification using local model networks," IEEE Trans. Power Syst., vol. 28, no. 3, pp. 2872-2881, Aug 2013.

[8] Konstantinos S. Metallinos, Theofilos A. Papadopoulos, and Charalambos A. Charalambous, "Derivation and evaluation of generic measurement-based dynamic load models," Elect. Power Syst. Res., vol. 140 , pp. 193 - 200, 2016.

[9] P. Jazayeri, W. Rosehart, and D. T. Westwick, "A multistage algorithm for identification of nonlinear aggregate power system loads," IEEE Trans. Power Syst., vol. 22, no. 3, pp. 1072-1079, Aug 2007.

[10] E. O. Kontis, T. A. Papadopoulos, A. I. Chrysochos, and G. K. Papagiannis, "Measurement-based dynamic load modeling using the vector fitting technique," Trans. Power Syst., Early Access.

[11] H. Bai, P. Zhang, and V. Ajjarapu, "A novel parameter identification approach via hybrid learning for aggregate load modeling," IEEE Trans. Power Syst., vol. 24, no. 3, pp. 1145-1154, Aug 2009.

[12] D. Karlsson and D. J. Hill, "Modelling and identification of nonlinear dynamic loads in power systems," IEEE Trans. Power Syst., vol. 9, no. 1, pp. 157-166, Feb 1994.

[13] D. J. Hill and I. A. Hiskens, "Dynamic analysis of voltage collapse in power systems," in Decision and Control, 1992., Proceedings of the 31st IEEE Conference on, 1992, pp. 2904-2909 vol. 3.

[14] D. J. Trudnowski and J. W. Pierre, "Overview of algorithms for estimating swing modes from measured responses," in 2009 IEEE Power Energy Society General Meeting, July 2009, pp. 1-8.

[15] T. A. Papadopoulos, E. O. Kontis, P. N. Papadopoulos, and G. K. Papagiannis, "System identification techniques for power systems analysis using distorted data," in MedPower 2014, Nov 2014, pp. 1-7.

[16] B. Gustavsen and A. Semlyen, "Rational approximation of frequency domain responses by vector fitting," IEEE Trans. Power Del., vol. 14, no. 3, pp. 1052-1061, Jul 1999

[17] B. Gustavsen, "Improving the pole relocating properties of vector fitting," IEEE Trans. Power Del., vol. 21, no. 3, pp. 1587-1592, July 2006.

[18] T. A. Papadopoulos, A. I. Chrysochos, E. O. Kontis, and G. K. Papagiannis, "Ringdown analysis of power systems using vector fitting," Elect. Power Syst. Res., vol. 141, pp. 100 - 103, 2016.

[19] T. A. Papadopoulos, A. I. Chrysochos, E. O. Kontis, P. N. Papadopoulos, and G. K. Papagiannis, "Measurement-based hybrid approach for ringdown analysis of power systems," IEEE Trans. Power Syst., vol. 31, no. 6, pp. 4435-4446, Nov 2016.

[20] "Load representation for dynamic performance analysis of power systems," IEEE Trans. Power Syst., vol. 8, no. 2, pp. 472-482, May 1993.

[21] J. V. Milanovic, K. Yamashita, S. M. Villanueva, S. Z. Djokic, and L. M. Korunovic, "International industry practice on power system load modeling," IEEE Trans. Power Syst., vol. 28, no. 3, pp. 3038-3046, Aug 2013. 